\title{
HST-FGS ASTROMETRY OF THE LOW MASS BINARY L722-22
}

\author{
L. G. TAFF \\ Department of Physics and Astronomy, The Johns Hopkins University \\ 3400 North Charles Street, Baltimore, MD 21218 U.S.A.
}

and

JOHN L. HERSHEY

Astronomy Programs, Computer Sciences Corporation, Space Telescope Science Institute, 3700 San Martin Drive, Baltimore, MD 21218 U.S.A.

The M dwarf L722-22 (= LHS 1047) was discovered to be a binary system by Ianna 20 years ago. The analysis of the ground- based data indicated a mass $0.06 M_{\odot}$ for the secondary. This is below the nominal stellar mass limit of $0.08 M_{\odot}$. The importance of potential "brown-dwarf" candidates, and the fact that the masses of both components place them near the end of the main sequence, made this system a prime object for further, intensive, study.

This close (separation $0 . " 3)$, faint $(V=11 . \mathrm{m} 5,14 . \mathrm{m} 4)$ binary was near the limit for ground-based work. The residuals of an individual night's photographic data were typically at the $50 \%$ level. Also, the photographic images are completely blended. The few one-dimensional speckle data points yielded a merged, asymmetric image profile. Finally, this system is too faint for HIPPARCOS. Our proposal for Hubble Space Telescope Fine Guidance Sensor (FGS) observing was approved in 1992.

By using the two FGS observing modes in tandem-"TRANSfer" mode to determine the separation of the pair projected onto the plane of the sky and "POSition" mode measurements to ascertain the trigonometric parallax and the motion of the primary about the center-of-mass of the system - the relative orbit and the fractional masses can be determined. Our combined use of the full facilities of the FGSs has become the standard binary star observing methodology. Anticipating this, and coupled with the importance of low mass objects near the end of the main sequence, our original HST observing proposal included a list of the six most promising systems possibly containing a sub-stellar member. All the others were subsequently awarded to other observers.

The relative orbital motion of the binary has been observed utilizing the astrometer FGS in its interferometric TRANS mode with the F583W filter. Such data can be rectified to within a milli-arc second (mas) or less and provides the angular separation of the components in two orthogonal dimensions. The absolute parameters of the orbit were determined by analyzing FGS POS mode measurements. The repeatability of this kind of observation is significantly worse, typically at the 3 mas level. Finally, the sky surrounding L722-22 is relatively empty. This makes the transformation from relative to absolute parallax uncertain.

Further details on the electro-optical characteristics of the FGSs and the reduction of TRANS and POS mode data are already published, by us, elsewhere. The 

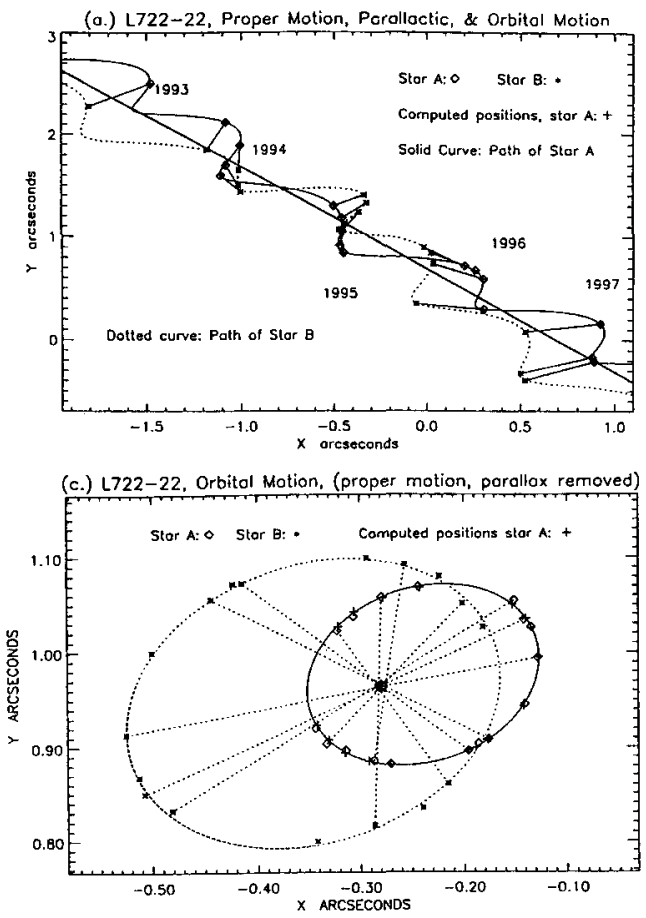

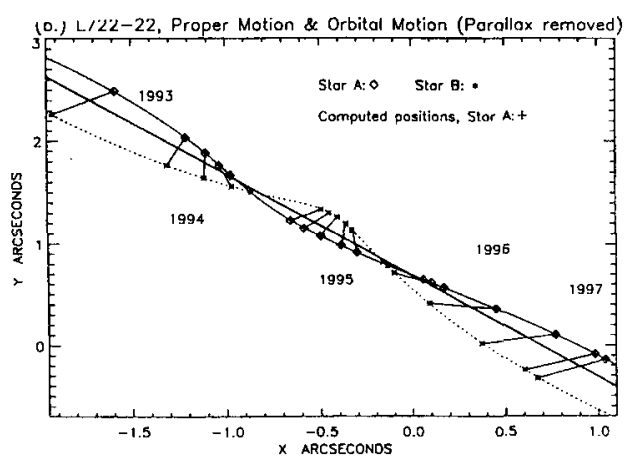

(d.) L722-22, Parallactic motion of STAR A

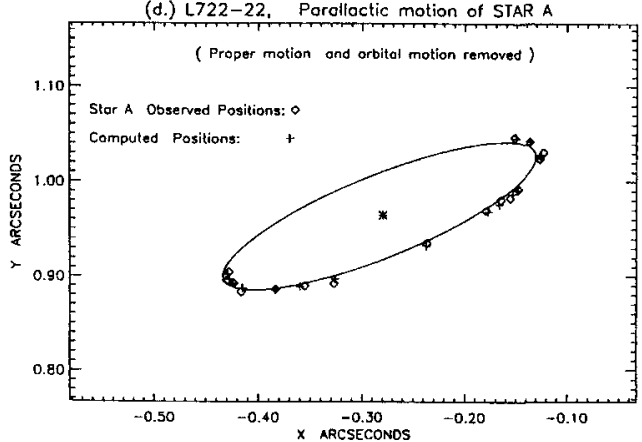

Fig. 1. The four figures show various view of the motions of the binary on the plane of the sky. In addition to the orbital motion, the binary has noticeable proper motion compounded by its path along its parallactic ellipse.

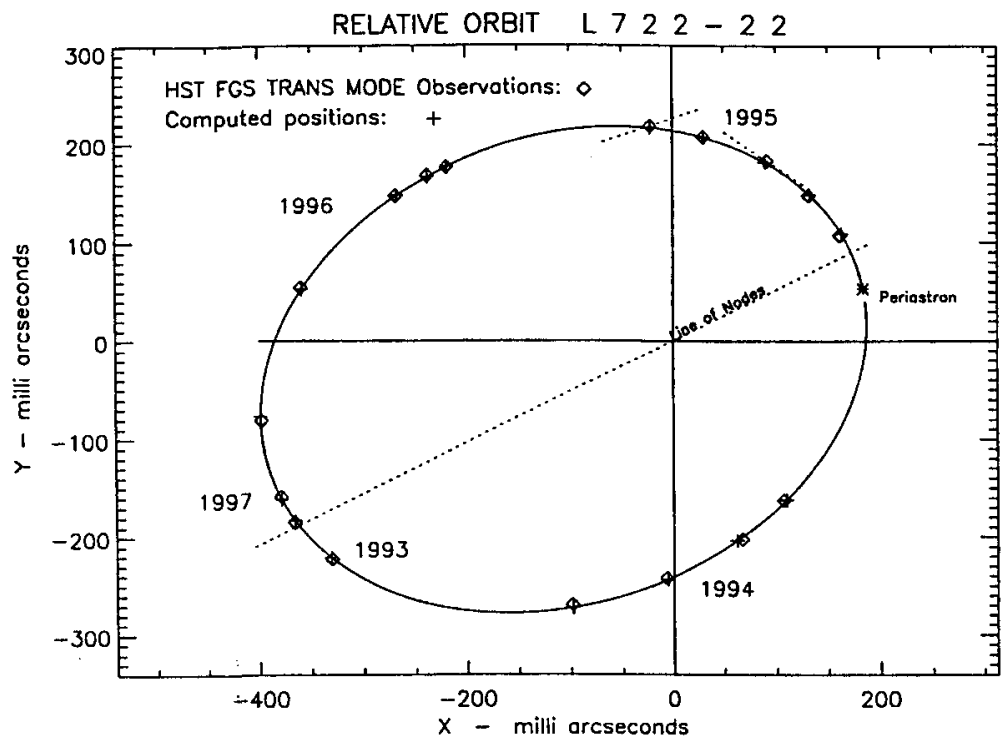

Fig. 2. Relative orbit of the binary. 
relatively red color of the system, B - V $=1 . \mathrm{m} 74$ required the use of an appropriately red calibration star. (See our forthcoming Astron. J. paper for the complete details and references.)

One can analyze TRANS mode measurements in one of two ways: by fitting the "sum" of two single star Transfer Functions to match the observed result or by Fourier transform de-convolution. We employed both procedures and they consistently provided the same results for the separations of the pair and the magnitude difference. We also utilized two methods to determine the relative orbit-both the classical orbital element set $(P, T, e, a, i, \omega, \Omega)$ and the Thiele-Innes constants were obtained. (The astrometric reduction of the POS mode data proceeded using standard STScI processing methods.) The results from our analysis, for this relatively eccentric orbit, are contained below along with the kinematic parameters.

\section{Classical Orbital Elements:}

$$
\begin{array}{ll}
P=4.566 \pm 0.009 \mathrm{yr} & T=1995.366 \pm 0.003 \mathrm{yr} \\
a=303.7 \text { mas } \pm 0.7 \text { mas } & e=0.364 \pm 0.001 \\
i=146.0 \pm 0.3(J 2000.0) & \omega=62.6 \pm 0.6(J 2000.0) \\
\Omega=-13.6 \pm 0.6(J 2000.0) &
\end{array}
$$

\section{Thiele-Innes Constants:}

$$
\begin{aligned}
& A=0 . " 0831 \pm 0 . " 0010 \quad B=0 . " 2894 \pm 0 . " 0007 \\
& F=0 . " 2522 \pm 0 . " 0007 \quad G=-0 . " 0492 \pm 0 . " 0015
\end{aligned}
$$

\section{Kinematic Parameters:}

$$
\begin{array}{ll}
\Pi_{\mathrm{rel}}=0 . " 1656 \pm 0 . " 0008 & \Pi_{\mathrm{abs}}=0 . " 1666 \\
\mu_{x}=0 . " 6009 / \mathrm{yr} \pm 0 . " 0006 / \mathrm{yr} & \mu_{y}=-0 . " 5993 / \mathrm{yr} \pm 0 . " 0005 / \mathrm{yr}
\end{array}
$$

The analysis of the 17 sets of FGS data, over nearly a complete orbital period (8/93 to 12/97), definitely eliminates the possibility that the secondary component has a sub-stellar mass. The values we found are $0.179 M_{\odot}$ and $0.112 M_{\odot}$ with a (formal) random error of only $1.5 \%$.

This work has been based on observations with the NASA/ESA HST obtained at the STScI which is operated by the AURA, Inc. under NASA contract NAS526555. Support for this work was provided, in part, by NASA through grant numbers $4283,5510,6063$, and 6641 from the STScI. 